\title{
QUALITY OF BASIC HEALTH SERVICES IN GOVERNANCE PERSPECTIVE
}

Oleh: Shermina Oruh: shoruh68@gmail.com; Andi Agustang: andi.agustang@unm.ac.id

\begin{abstract}
The application of good governance within governance prerequisite that cannot be left out is the participation of the community in public policy. The main prerequisites were when the Government implemented the autonomous region with the principle of decentralization, community participation. It involves all aspects of the implementation of the development in areas starting from planning to supervision. Participatory governance is governance putting citizens or non-government as an individual or organization as a viable social stakeholder in making public policy that has just dominated the Government. Model of participatory governance policy in the primary health services is eligible to be developed by optimizing and strengthening cooperation intersectional, increasing the motivation of health workers as well as eliminating social and cultural barriers in the community.
\end{abstract}

Keywords: Participatory governance, primary health services

\section{INTRODUCTION}

There is an understanding that the community is the owner of the public service. Therefore, the community should be included in making decisions related to the implementation of the public service. There will be many advantages by engaging the community in decision making related to public service. There is an increase in the quality of the resulting public policy the Government that in turn will be very beneficial to the community as a policy goal. In addition to creating benefits for the community, public participation in decision-making will also provide benefits for the Government. It means that the government will be more powerful in the institutional capacity in the presence of policy produced democratically.

The community participation in the public service is crucial. Therefore, the community could play a role such as formulating criteria for service, learning how to set up the service, granting each engagement, adjusting the mechanism of public complaints and monitoring as well as evaluating the implementation of the public service. Thus, the Government andthe community can jointly build commitment to creating quality public services. 
The first barrier is the problem of laziness to participating in research conducted by Abelson and Elsey (1998) [1] concluding that in the end, there is a tendency of participation that is issue-issued. It means that the community will participate in the simple problems by not using a high analytical and theoretical ability toward a problem. Another barrier is the problem of the implementation of exit and voice within the public service, as revealed by Alberth Hiischman in Kurniawan (2008) [2]. He states that the exit mechanism is usually hampered by expensive service provision so that the citizens are reluctant to do that. The barrier on the mechanism of voice is the absence of a channel for it. These phenomena show that the participation of the community in the public service is still poor.

\section{THEORETICAL FRAMEWORK}

The concept of Participatory Governance

The concept of participatory governance in social sciences has prompted a new philosophy to develop this concept from a broader perspective. Geissell (2004) [3] in his article states: "Participatory Governance Theoretic-analytical Approaches and a Case Study (Traditional Network)" The models of participatory governance are identified into three perspectives. They are global governance, participatory governance, and participatory governance in non-state transnational networks.

From the above description, it can be concluded that participatory governance is a Government putting the citizens or non-government as an individual or organization for social development as stakeholders in decision making for public policy that dominated the government. Regardless of whether the Government is using a system of direct democracy or indirect democracy or by mixing the two systems of democracy. Citizen participation at the level of concepts, as well as practical studies, can be created without regard to any democratic system that is used. Thus, from this perspective, participation is no longer understood as a means or method, but it is understood as a process and goal.

The service quality is the difference between the perception and experience of consumers, who state an allegation of satisfaction. Whether the public service has a high quality or not, can be understood as a disproportionate gap both in public organizations are recognized (Parasuraman et al., 1985). [4] 
According to Grönroos (1990),[5] there are two types of service quality, namely: First, the technical quality / technical quality (outcome dimension) related to the perceived output quality customer service. The components are divided into three types, namely: search quality (can be evaluated before purchase, such as price), quality experience (can only be evaluated after consumption, e.g. punctuality, speed of service and neatness results), and Credence quality (though a difficult customer has evaluated consume services, such as the quality of cardiac surgery). Second, the quality of the functional / functional quality (process-related dimension) about the quality of service delivery means or involves the transfer of technical quality, outputs or outcomes from the service provider to the customer. It is examples of the appearance and behavior of the waiter. When compared with the technical quality, functional quality dimension is perceived subjective and could not be evaluated as objectively as technical quality.

Participatory Governance policy models in health care deserve to be developed (Pollit, 1999) [6] is due to: 1) transaction that occurs between providers and users of services in the public sector tend to be more complex than those faced by service users in a normal market. 2) The consumer sector public that they are not just consumers, but it is also a citizen of course it has certain unique implications. Hospital as a health care provider institution also still needs to develop appropriate service quality expectations of the customer.

In Service Quality Conceptual Model, there are five services prose possibility of a gap that causes the difference in perception of service quality. Quality of care is the degree of concordance between the expectations / desires and perceptions of service received by the customer. There needs to be an effort to eliminate the differences in perception (gap) on the quality of service of the provider to the customer. As the effort is to improve the suitability on meeting the needs and expectations of the various dimensions of service quality. Fulfillment waiter is quality dimensions according to the needs and expectations of the community will provide a positive perception of the quality of services provided.

Public Service

Service is a translation of the word "service" is often translated into services. According to Kotler (1995) [7], the service is any action or activity that can be offered by one party to another that is essentially intangible and does not result in any ownership.

Public services can be defined; any services performed by the Central Government, in the region, and the State-Owned Enterprises environment or provincial enterprises in the form of goods or services, either in an effort to meet the needs of society as well as in the implementation of the laws and invitation (Decision MENPAN Number 63/2003). 
In connection with the assessment of service quality Morgan and Murgatroyd (1994) [8] The portrait of the Triangle of Service Quality. The costumer loyality, the future of hospitality marketing Lewis (1999) [9]

As for The Triangle of Quality Service, is as follows:

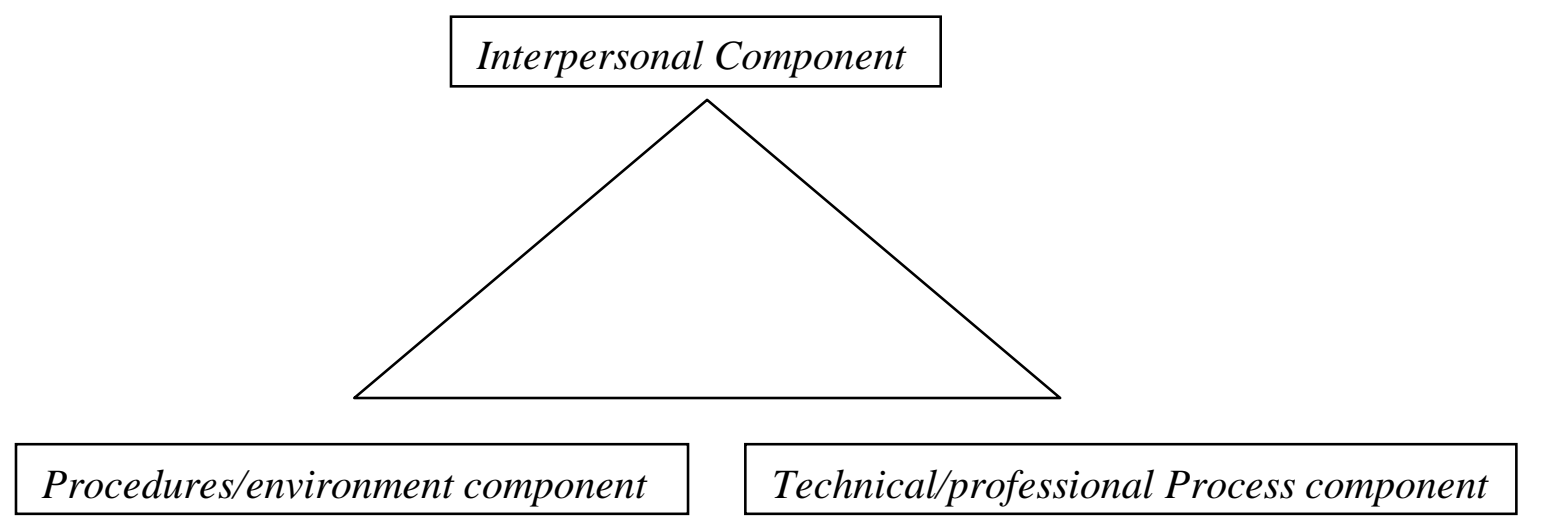

Figure 1. The Triangle of Service Quality

Source: Warella (2004:74) tahun 1997[10]

This model is an equilateral triangle where the peak is a component of Interpersonal. Of a service, whereas on the left side of the triangle is the physical context, procedures and process components. On the right, side is the technical or professional component in delivering the service. The assumption of this model is the need for sustained balance of these three components in providing a good service. For example, too much emphasis on process and procedure will give the impression that services convoluted and complicated.

\section{Basic Health Care}

Public Service Health Division has been supported by Law No. 23 in 1992 on Public Health Systems. This law has set a range of issues related to health problems, both of which include philosophy, arrangement between rights and obligations, duties and responsibilities, efforts and human health. The law has established that health development aims to increase awareness, willingness and ability of healthy life for every person to realize the optimal degree of public health.

Everyone has equal rights in obtaining optimal health status (Article 4), and the government is responsible for improving public health (Article 9). Under this law, health efforts include: Family Health, Nutrition repairs, Security Food and Drink, Environmental Health, Occupational Health, Mental Health, Communicable Disease, Illness and Healing Health 
Recovery, Health Education, Safety of Pharmaceutical and Medical Devices, Security Addictive substances, School of Health, Exercise Health, Traditional Medicine, Eye Health

\section{DISCUSSION}

Paradigm shift of public service that leads to New Public Service (NPS) has an impact on government bureaucracy to encourage public participation in the public service. So also in the basic services of health conducted by local governments do positive measures to encourage participation by the community are expected to not only the utilization of service but participated in the planning process service.

With respect to the health service in Maros has sought attractive businesses with different forms of community participation to regard the basic services of health, namely:

a. Socialize the Local Regulation No.7 in 2011, it is about health care Levying on health services with the aim of the community to know the costs incurred by the community in order to get health care at Clinics and laboratories with the kind of services that are quite diverse.

b. The perform of health promotion in the interactive dialogue, creation of mass communication, information and education (posters, leaflets, and turning sheet), a public service advertisement creation through print and electronic media, radio broadcasts, interactive dialogue through TV and radio, road show of health/exhibition clinics in the region health services in Maros.

c. Close to the access for medical services through mobile Clinics

d. Through Alert Village/Health Village Post

e. Improvement of quality of service clinics through international standardization of quality of service. (ISO 9001:2008). 
If the described model findings to medical services from the perspective of Participatory Governance is as follows:

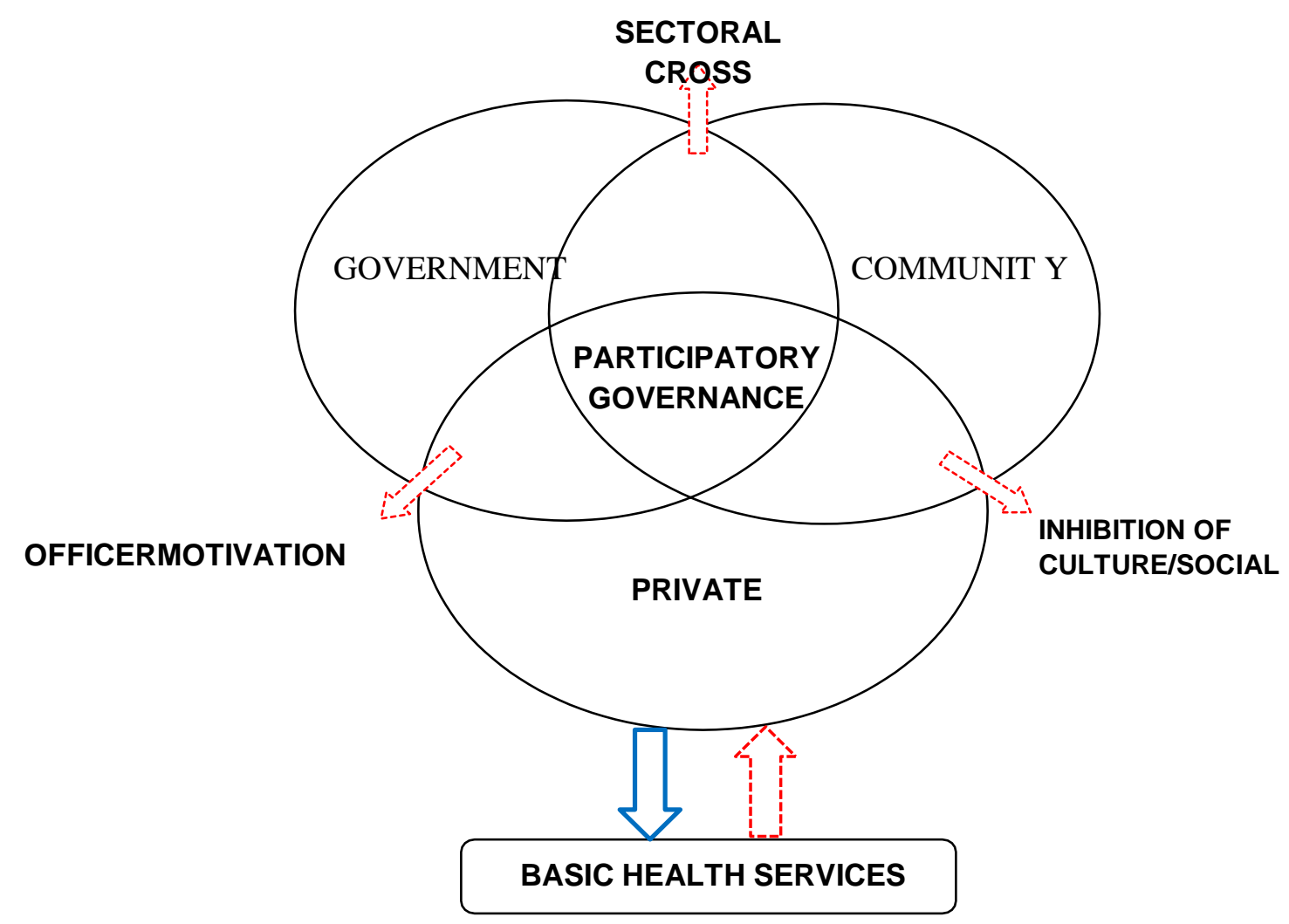

Figure 2. The Findings of the Health Care Model from the Perspective of Participatory Governance

Models of health care from the perspective of Participatory Governance, namely: factually, field conditions associated with health care from the perspective of Participatory Governance gives the explanation that the Participatory Policy Governance Model in the basic services of health deserves to be developed by optimizing as well as strengthen between cooperation and intersectional. The increasing the motivation of health workers as well as eliminate social barriers and cultures in the community.

\section{CONCLUSION}

The empirical basis of this research found several things that can develop community participation in the basic services of health, namely:

1. In order to develop community participation in the basic services of health then, the organizer provides the space to provide opinions and proposals on the problems community 
face, namely through musrenbang, Alert Village and Health Care Forum (FDK/ Forum Peduli Kesehatan) clinic.

2. The organizer provides a clear space to the community to convey complaints directly or indirectly either via the suggestion box or other media (SMS, Fax, telephone, others) so that the community felt it had been granted the right as the owner of the public service.

3. Community involvement in the evaluation process should be through the mechanism of Community Satisfaction Index rating (IKM/ Indeks Kepuasan Masyarakat) that can be relied upon to service improvement in accordance with the expectations of society.

\section{REFERENCE}

Albelson, J and Eyles, 1998. Public Participation and Citizen Governance in Canadian Health System Discussion Paper No.7 Commision On The Future of HealthCare in Canada

Kurniawan, Luthfi J dan Mokkhamad Najib, Paradigma kebijakan Pelayanan Publik. In-Trans Publishing Malang.

Geissel, Brigette, 2004. Participatory Governance Theoritic-analytical Approaches And A case Study, Fifth Pan-Europen International Relations Conference The Hague, September 911

Parasuraman, A, Zeeithaml, V.A. and Berryl. LL, 1985, “ A Conceptual Model Of Service Quality and Its Implication for Future Research," Journal of Marketing Retailing, Vol 49 No.4 : ABI/INFORM Global.

Gronroos, C. (1990). "Relationship Approach to marketing in service Contexts : The Marketing and Organizational Interface", Journal of Business Research, Vol. 20 (January) , pp 3-11

Pollitt, C. (1999) "Stunded By Stake Holders ? Limits to Collaborate', Public Policy and Administration.

Kotler, P and Andewreasen. 1991. St Rategic Marketing for Non Profit Organization. Eglewood Cliffs, NJ : Preentise Hall

Morgan Collin and Murgatroyd Stephen, 1994, Total Quality Management in the Public Sector. Open University Press.

Lewis C. Robert. Shoemaker Stowe. 1999, “ Customer loyality: The Future of Hospitality Marketing “. International Journal of Hospitality Management 18 (1999) 345-370

Warella, Y. (1997) Administrasi Negara dan Kualitas Pelayanan Publik, Pidato Pengukuhan Guru Besar, FISIP UNDIP

\section{Sumber Inspirasi}

Agustang, A. D. M. P., \& Agustang, A. (2021, December 15). Studi Politik Ekonomi Dalam Permulaan Wacana Development. https://doi.org/10.31219/osf.io/mx8gp

Tinri, M. D. N. (2018, June 26). Hotels and Restaurant Tax Revenues Management. https://doi.org/10.17605/OSF.IO/B8W7J 
Agustang, A. (2021, January 10). INTERAKSI SOSIAL KOMUNITAS LOKAL DENGAN PENDATANG DAN PERUBAHAN STRUKTUR KOMUNITAS LOKAL (Studi pada Masyarakat Majemuk di Kawasan Industri Makassar). https://doi.org/10.31219/osf.io/pw5xr

Agustang, A., Ariani, A. I., \& Asrifan, A. (2021, January 10). KONSTRUKSI SOSIAL JANDA TEREKSPLOITASI (STUDI KASUS DI KELURAHAN TETEBATU KECAMATAN PALLANGGA KABUPATEN GOWA). https://doi.org/10.31219/osf.io/ryz2k

Agustang, A. (2021, January 22). Filosofi Research Dalam Upaya Pengembangan Ilmu. https://doi.org/10.31219/osf.io/9n6za

Kurniawan, A., \& Agustang, A. (2022, January 11). FAKTOR PENGHAMBAT TINGKAT KEDISIPLINAN SISWA DI SMAN 1 BANTAENG. https://doi.org/10.31219/osf.io/qnx7z

Lestari, S. A., \& Agustang, A. (2022, January 11). EFEKTIVITAS PEMBELAJARAN ONLINE PADA MATA PELAJARAN SOSIOLOGI DI SMA NEGERI 22 MAKASSAR. https://doi.org/10.31219/osf.io/pgw8t

Quraisy, H., Agustang, A., \& Asrifan, A. (2022, January 11). MUHAMMADIYAH DALAM GERAKAN SOSIAL DI KABUPATEN WAJO. https://doi.org/10.17605/OSF.IO/ZWDN6

Quraisy, H., Agustang, A., \& Asrifan, A. (2021, January 15). MUHAMMADIYAH DALAM GERAKAN SOSIAL DI KABUPATEN WAJO. https://doi.org/10.31219/osf.io/dpsg9

Oruh, S., Agustang, A., \& Asrifan, A. (2021, January 9). PENGARUH PENGETAHUAN KELUARGA, STIGMA MASYARAKAT DAN KEPATUHAN MINUM OBAT TERHADAP KEKAMBUHAN PENYAKIT GANGGUAN JIWA DI KOTA MAKASSAR. https://doi.org/10.31219/osf.io/wxsg8

Agustang, A., \& Oruh, S. (2021, January 22). Teknologi Partisipasi Metode Fasilitasi Pembuatan Keputusan Partisipatif. https://doi.org/10.31219/osf.io/dhfb7

Oruh, S., Agustang, A., \& Theresia, M. (2021, January 9). KESEJAHTERAAN PSIKOLOGIS (Studi Pada Dewasa Madya yang Belum Menikah di Kota Makassar). https://doi.org/10.31219/osf.io/v5af8

S., Agustang, A. D. M. P., \& Agustang, A. (2022, January 9). DINAMIKA PUTUS SEKOLAH DI KALANGAN PEKERJA ANAK DI KOTA MAKASSAR. https://doi.org/10.31219/osf.io/ucbz4

Agustang, A., Oruh, S., \& Mahmuddin, H. (2022, January 4). IMPROVING THE QUALITY OF PRIMARY HEALTH SERVICES IN THE PERSPECTIVE OF PARTICIPATORY GOVERNANCE. https://doi.org/10.17605/OSF.IO/9T8VQ

Oruh, S. (2022, January 4). STRATEGI PENGEMBANGAN SUMBER DAYA MASYARAKAT KEPULAUAN YANG UNGGUL MENUJU SOCIETY 5.0. https://doi.org/10.17605/OSF.IO/SP42D

Oruh, S. (2022, January 4). Supplemental materials for preprint: KAU MAU KEMANA (Refleksi Sosiologis terhadap Integritas Upaya Kesehatan Jiwa). https://doi.org/10.17605/OSF.IO/CP39Z

Oruh, S., \& Agustang, A. (2022, January 4). KESEJAHTERAAN PSIKOLOGIS (Studi Pada Dewasa Madya yang Belum Menikah di Kota Makassar). https://doi.org/10.17605/OSF.IO/ZUSKV

Oruh, S., \& Agustang, A. (2022, January 4). PENGARUH PENGETAHUAN KELUARGA, STIGMA MASYARAKAT DAN KEPATUHAN MINUM OBAT TERHADAP KEKAMBUHAN PENYAKIT GANGGUAN JIWA DI KOTA MAKASSAR. https://doi.org/10.17605/OSF.IO/K4VMP

Agustang, A., \& Oruh, S. (2022, January 4). HEGEMONIC SOCIAL RELATION A Study of Sociology on Outsourcing Practice. https://doi.org/10.17605/OSF.IO/PJM3A

Oruh, S., Agustang, A., \& Agustang, A. D. M. P. (2021, December 21). SOCIAL DETERMINANTS OF COMMUNITY HEALTH WITH THE EVENT OF DIABETES MELLITUS (Study of Health Sociology in Makassar City). https://doi.org/10.31219/osf.io/4qvxf

Tilome, A. A., Agustang, A. D. M. P., \& Agustang, A. (2021, December 16). PERTUKARAN SOSIAL ELIT POLITIK DALAM PEMILIHAN KEPALA DAERAH DI PROVINSI GORONTALO. https://doi.org/10.31219/osf.io/6tu79 
Agustang, A., Ahriani, A., \& Asrifan, A. (2021, January 10). MARGINALISASI BUDAYA (STUDI PADA PRANATA SOSIAL MASYARAKAT MUSLIM SUKU KOKODA KOTA SORONG). https://doi.org/10.31219/osf.io/942y8

Agustang, A., A, A. D. M. P., \& Asrifan, A. (2021, January 20). PERAN PENDIDIKAN DALAM MEMBANGUN KARAKTER BANGSA. https://doi.org/10.31219/osf.io/na9pu

Quraisy, H., Agustang, A., \& Asrifan, A. (2021, January 15). MUHAMMADIYAH DALAM GERAKAN SOSIAL DI KABUPATEN WAJO. https://doi.org/10.31219/osf.io/dpsg9

Agustang, A. (2021, January 10). KERAJINAN TENUN PADA MASYARAKAT MUNA (Kasus Peranan Modal Manusia dan Modal Sosial Dalam Reproduksi Budaya Tenun di Kabupaten Muna). https://doi.org/10.31219/osf.io/4bksf

Agustang, A., \& Oruh, S. (2021, January 22). Teknologi Partisipasi Metode Fasilitasi Pembuatan Keputusan Partisipatif. https://doi.org/10.31219/osf.io/dhfb7

Agustang, A. (1999). INTERAKSI SOSIAL DAN PERUBAHAN STRUKTUR KOMUNITAS. Universitas Padjadjaran Bandung.

Agustang, A. (2009). SIMBOLIKHAJI: Studi Deskriptif Analitik pada Orang Bugis. Al-Qalam, 15(2), 317-334.

Agustang, A. (2014). LIFE BEHAVIOR OF SOCIETY, FIVE CASES ON FAMILY OF DIABETES MELLITUS PATIENTS IN MAKASSAR. International Journal of Academic Research, 6(1).

Agustang, A. (2018). PERILAKU MEROKOK REMAJA SISWA SMA NEGERI 1 SINJAI UTARA KABUPATEN SINJAI. Jurnal Ilmiah Kesehatan Diagnosis, 13(1), 93-99.

Agustang, A. (2021). Teknologi Partisipasi Metode Fasilitasi Pembuatan Keputusan Partisipatif. OSF Preprints.

Agustang, A., \& Adam, A. (2020). INSTITUTIONAL FAILURES IN THE WORLD OF EDUCATION IN SCHOOLS FOR CHILDREN VICTIMS OF PARENTS. Asian Journal of Social Sciences \& Humanities, 9(2), 18-26.

Agustang, A., \& Indah, A. M. (2021). Andi Asrifan. 2021.“. KONSTRUKSI SOSIAL JANDA TEREKSPLOITASI (STUDI KASUS DI KELURAHAN TETEBATU KECAMATAN PALLANGGA KABUPATEN GOWA). " OSF Preprints. January, 10.

Agustang, A., \& Mustafah, M. Y. (2017). METROSEKSUAL DIKOTA MAKASSAR.

Agustang, A., \& Oruh, S. (2021). KESEJAHTERAAN PSIKOLOGIS (studi Pada Dewasa Madya Yang Belum Menikah Di Kota Makassar).

Agustang, A., Saleh, S., Rasyid, R., \& Agustang, A. T. P. (2019). Pengembangan profesionalisme guru SMP Negeri 1 Duampanua Kabupaten Pinrang melalui pelatihan penulisan karya tulis ilmiah. Seminar Nasional Pengabdian Kepada Masyarakat, 2019(2).

Agustang, A., Suardi, S., Putra, A. D. M., \& Oruh, S. (2021). Pemberdayaan Guru Mata Pelajaran Sosiologi Melalui Literasi Digital Berbasis Quick Response Code di Kecamatan Bissappu Kabupaten Bantaeng. Abdi: Jurnal Pengabdian Dan Pemberdayaan Masyarakat, 3(2), 175188.

Agustang, A. Y. A., Herman, H., Said, M., \& Agustang, A. (2021). Upaya Guru IPS Dalam Meningkatkan Motivasi Belajar Siswa Pada Masa Covid 19 Di SMP. Phinisi Integration Review, 4(1), 144-149.

Ahriani, A., Agustang, A., Adam, A., \& Upe, A. (2020). The Multiple Roles of Women in Poor.

Akbar, H., Oruh, S., \& Agustang, A. (2021). Indeks Prediktif Kejadian Demam Berdarah Dengue 
(DBD) Berbasis Perilaku Sosial Masyarakat Di Kabupaten Indramayu. Jurnal Kesehatan, 14(2 SE-), 76-82. https://doi.org/10.32763/juke.v14i2.289

Al Yakin, A., \& Agustang, A. (2020). SELEBRIFIKASI DAN ELEKTABILITAS; MENAKAR RESPON PUBLIK.

Alim, A., Agustang, A., \& Adam, A. (2021). Transformation of Consumption Behavior of the Poor in the Case of Malnutrition: Health Sociology Study with Participatory Approach in Makassar City, Indonesia. Open Access Macedonian Journal of Medical Sciences, 9(E), 598-607.

AM, S. A., Agustang, A., Mustadjar, M., \& Tahmir, S. (2020). Mobility and Social Change of the Economy of Indonesian Migrant Workers in Indonesia. Solid State Technology, 63(5), 408417.

Aminah, S., Agustang, A., Idkhan, A. M., \& Rifdan, R. (2021). KUALITAS PELAYANAN PUBLIK PADA KANTOR PERTANAHAN KABUPATEN PANGKAJENE DAN KEPULAUAN. JISIP (Jurnal Ilmu Sosial Dan Pendidikan), 5(4).

Amran, N. G., \& Agustang, A. (2020). Implementasi Kurikulum 2013 Dalam Peningkatan Kompetensi Psikomotorik Dan Afektif Siswa. Phinisi Integration Review, 3(2), 179-184.

Andriana, Agustang, A., Idkhan, A. M., \& Hasriadi. (2021). Daya Tanggap Pelayanan Unit Gawat Darurat di Puskesmas Sarjo Kabupaten Pasangkayu . Journal Governance and Politics (JGP), 1(2 SE-Articles). https://e-journal.iyb.ac.id/index.php/jgp/article/view/201

Ardiansyah, A. A., \& Agustang, A. (2018). MAHASISWI BERKELUARGA DI FAKULTAS ILMU SOSIAL UNIVERSITAS NEGERI MAKASSAR. JURNAL SOSIALISASI, 19-23.

Aris, Agustang, A., \& Idkhan, A. M. (2021). Efektifitas Pelayanan Aparatur Sipil Dalam Meningkatkan Pelayanan Publik. Journal Governance and Politics (JGP), 1 (2 SE-Articles). https://jurnal.iyb.ac.id/index.php/jgp/article/view/208

Arisal, A., Agustang, A., \& Syukur, M. (2020). Diskriminasi Sosial Anak Penderita Kusta di Kota Makassar. Phinisi Integration Review, 3(2), 297-304.

Arpin, Agustang, A., \& Idkhan, A. M. (2021). Pengaruh Penerapan Model Dicovery Learning Terhadap Hasil Belajar Siswa Pada Mata Pelajaran IPA SD Inpres Tamalanrea V Kota Makassar. Journal Governance and Politics (JGP), 1(2 SE-Articles). https://ejournal.iyb.ac.id/index.php/jgp/article/view/204

Arwin, Agustang, A., Ihsan, A., \& Suhaeb, F. (2015). POVERTY AND MODERNIZATION IN BAJO FISHING COMMUNITY. International Journal of Academic Research, 7(Vol. 7. No. 3. May, 2015).

Asri, A., Kasnawi, T., \& Agustang, A. (2019). Actors' Social Tensions on Traditional Birth Attendants and Midwife Partnerships in Childbirth Assistance (Sociology of Health Study in Bulukumba Regency). IOSR Journal Of Humanities And Social Science (IOSR-JHSS), 24(Issue 1, Ser. 10), 20-27.

Aswan, A., \& Agustang, A. (2017). PERILAKU ANOMI (STUDI PADA SISWA DI SMP NEGERI 21 MAKASSAR). JURNAL SOSIALISASI, 62-66.

Azis, F., Idris, R., \& Agustang, A. (2017). Involution Fisheries: Post-Study Model of National Program in Integrated Movement at Community Fishermen's Village Development Arungkeke, Jeneponto. Mediterranean Journal of Social Sciences, 8(1 SE-Articles), 441. https://www.richtmann.org/journal/index.php/mjss/article/view/9713

Badwi, A., Agustang, A., \& Adam, A. (2018). Individual Cognitive Dissonance in Society on Condom (A Sociology of Health Study in Makassar City). IOSR Journal Of Humanities And 
Social Science (IOSR-JHSS), 23(Issue 8, Ver. 7), 14-19.

Badwi, A., Agustang, A., \& Adam, A. (2020). Individual Cognitive Dissonance in Society on Condom (A Sociology of Health Study in Makassar City).

Bastiana, B., Jumadi, J., Herman, H., \& Agustang, A. D. M. P. (2019). Pelatihan penulisan Penelitian Tindakan Kelas bagi guru SMP Negeri 1 Duampanua Kabupaten Pinrang. Seminar Nasional Pengabdian Kepada Masyarakat, 2019(2).

Bebang, M., \& Agustang, A. (2021). THE SOCIAL ACTION OF THE TEACHERS IN HANDLING INDISIPLINARY STUDENTS AT SMP NEGERI 36 MAKASSAR, SOUTH SULAWESI, INDONESIA. 20(5), 5792-5796. https://doi.org/10.17051/ilkonline.2021.05.652

Bebang, M., Agustang, A., \& Samad, S. (2021). THE SOCIAL ACTION OF THE TEACHERS IN HANDLING INDISIPLINARY STUDENTS AT SMP NEGERI 36 MAKASSAR, SOUTH SULAWESI, INDONESIA. Elementary Education Online, 20(5), 5792-5796.

Bintaha, M., Salman, D., Agustang, A., \& Suhaeb, F. W. (2014). THE ROLE OF SOCIAL CAPITAL TOWARD SOME OTHER CAPITALS FOR RURAL ADVANCEMENT. International Journal of Academic Research, 6(5).

Burchanuddin, A., Agustang, A., Adam, A., \& Alim, A. (2021). Cultural Reproduction in the Sociopolitical Context of Bone District, South Sulawesi, Indonesia.

Daming, M., Agustang, A., Idkhan, A. M., \& Rifdan, R. (2021). IMPLEMENTASI KEBIJAKAN MAKASSAR RECOVER DALAM PENANGANAN COVID-19 DI KOTA MAKASSAR. JISIP (Jurnal Ilmu Sosial Dan Pendidikan), 5(4).

Dirawan, G. D. (2015). FOSTERING COLLABORATION STREET CHILDREN IN MAKASSAR. Man In India, 95(3), 569-576.

Djalante, A., Agustang, A., Tahmir, S., \& Sahabuddin, J. (2018). A Disadvantaged Tribe in Bajoe Village. Bone Regency: A Phenomenological Research on Bajo Tribe's Social Life in Bone Regency, South Sulawesi.

Djamaluddin, M., Agustang, A., Idkhan, A. M., \& Rifdan. (2021). Pengaruh Implementasi Corporate Social Responsibility PT. Vale Terhadap Kesejahteraan Hidup Masyarakat. Journal Governance and Politics (JGP), $1(2$ SE-Articles). https://jurnal.iyb.ac.id/index.php/jgp/article/view/200

Edyanto, E., Agustang, A., Idkhan, A. M., \& Rifdan, R. (2021). Implementasi Kebijakan Otonomi Khusus (Otsus) Papua. JISIP (Jurnal Ilmu Sosial Dan Pendidikan), 5(4).

Firdaus, F. (2017). Permandian Wae Pellae (Konstruksi Fisik Spasial dan Perubahan Masyarakat Sinjai). Equilibrium: Jurnal Pendidikan, 5(1), 15-20.

Hairuddin, Agustang, A., Manda, D., \& Jumadi. (2018). Dynamics of Social Behavior of Using Condoms in Brothel at Jalan Nusantara, Makassar City (Sociology of Health and Illness Review). IOSR Journal Of Humanities And Social Science (IOSR-JHSS), 23(Issue 3, Ver. 11), 33-39.

Haniah, S., Agustang, A., \& Samad, S. (2014). SOCIAL ACTION IN THE CLASSROOM: ANALYSIS OF EDUCATIONAL SOCIOLOGY ON INTERACTION BETWEEN TEACHER AND STUDENT IN SMP KARTIKA CHANDRA MAKASSAR. International Journal of Academic Research, 6(4).

har, M., Agustang, A., Adam, A., \& Upe, A. (2021). Online Learning and Distortion of Character Education in the Covid-19 Pandemic Era. Webology, 18(Special Issue 04), 566-580. https://doi.org/10.14704/web/v18si04/web18149 
Hariani, H., Oruh, S., \& Mustari, N. (2021). GAMBARAN POLA ASUH ANAK PADA IBU YANG BEKERJA DI KELURAHAN KARANG ANYAR KOTA MAKASSAR. Media Keperawatan: Politeknik Kesehatan Makassar, 12(1), 1-6.

Harman, H., \& Agustang, A. (2020). PERUBAHAN SOSIAL MASYARAKAT PERMANDIAN WAE TUO DESA WAE TUO KECAMATAN KAJUARA KABUPATEN BONE. Jurnal Sosialisasi: Jurnal Hasil Pemikiran, Penelitian Dan Pengembangan Keilmuan Sosiologi Pendidikan, 2, 1-7.

Hermansya, H. (2014). Pengaruh kompetensi profesional guru sosiologi Terhadap Hasil Belajar Ssiswa di SMA Negeri 1 Soppeng Riaja Kabupaten Barru. Skripsi. Dibimbing oleh Prof. Dr. Andi Agustang, M. Si dan H. Dr Muhammad syukur, M, Si. Program Studi Pendidikan Sosiologi Fakulta. universitas negeri makassar.

Hikmah, N., \& Agustang, A. (2017). PERAN POST TEST TERHADAP MOTIVASI BELAJAR PADA MATA PELAJARAN SOSIOLOGI SISWA DI SMA NEGERI 11 MAKASSAR. JURNAL SOSIALISASI.

HIKMAH, N., \& AGUSTANG, A. (2016). PERAN POST TEST TERHADAP MOTIVASI BELAJAR SISWA PADA MATA PELAJARAN SOSIOLOGI DI SMA NEGERI 11 MAKASSAR. Universitas Negeri Makassar.

Idrus, I. I., Salman, D., \& Agustang, A. (2021). Commodification of Environmental Discourse in the Process of Producing Space in Bukit Baruga Housing, Makassar. IOP Conference Series: Materials Science and Engineering, 1125(1), 12087.

Irwan, A. A., Adam, A., \& Upe, A. (2021). Community Empowerment Strategy towards a Sustainable Rural Community-based Tourism Village. Turkish Online Journal of Qualitative Inquiry (TOJQI), 12(5), 2065-2076.

Ismanto, Agustang, A., \& Idkhan, A. M. (2021). Pengaruh Kompensasi Finansial Terhadap Produktivitas Kerja Karyawan Pada PT PLN (PERSERO) Rayon Makassar Barat. Journal Governance and Politics (JGP), $1(2 \quad$ SE-Articles). https://ejournal.iyb.ac.id/index.php/jgp/article/view/202

Jumadi, Nurlela, Agustang, A., \& Darman. (2020). Social Actions of Galla Actors in the Kaemba Maros Traditional Village Community Sulawesi Selatan. 3rd International Conference on Social Sciences (ICSS 2020), 748-753.

Jumharyanti, J., \& Agustang, A. (2018). REMITANSI TENAGA KERJA INDONESIA PASCA KEPULANGAN DARI LUAR NEGERI (STUDI TKI DI DESA WORO KECAMATAN MADAPANGGA KABUPATEN BIMA NTB). Jurnal Sosialisasi: Jurnal Hasil Pemikiran, Penelitian Dan Pengembangan Keilmuan Sosiologi Pendidikan, 68-73.

Kasnawi, T., \& Agustang, A. (2014). TRADITIONAL ELITE ON MODERN POLITICS AT SOUTH SULAWESI. International Journal of Academic Research, 6(4).

Khaerun, K. (2014). Khaerun. Pengaruh kemampuan Manajerial Kepala Madrasah dan Kompetensi Pedagogik terhadap Kinerja Guru di Madrasah Tsanawiyah Kabupaten Gowa Provinsi Sulawesi Selatan.(Dibimbing oleh Andi Agustang dan Jumadi Tangko). universitas negeri makassar.

Kubangun, N., Agustang, A., \& Adam, A. (2020). Subaltern and Network Strategy Towards Fishermen Community in Aru Islands District. 3rd International Conference on Social Sciences (ICSS 2020), 585-588.

LEGIYO, L., Agustang, A., \& Hakim, M. (2018). Relasi Punggawa dan Sawi pada Bagan Perahu (Studi Kasus Nelayandi Pulau Balang Lompo Kabupaten Pangkep). UNIVERSITAS 


\section{NEGERI MAKASSAR.}

Leiwakabessya, J. E. M., Kasnawi, M. T., \& Agustang, A. (2021). The reality of the conflict of customary lands between the land of laha and the air force in ambon city (case study customary land in the land of laha). Turkish Online Journal of Qualitative Inquiry (TOJQI), 12(09), 4797-4804.

Makmur, M. A., Agustang, A., \& Idkhan, A. M. (2021). Tingkat Kepuasan Masyarakat Terhadap Pelayanan Publik (Evaluasi Implementasi Program Beras Sejahtera Di Kota Palopo). Jurnal Sosio Sains, 7(2), 131-138.

Masita, M., \& Agustang, A. (2020). Pergeseran Pemaknaan Perayaan Tradisi Saiyyang Pattu'duq di Tinambung Polewali Mandar. Phinisi Integration Review, 3(2), 173-178.

Mufidah, N. F., \& Agustang, A. (2020). STUDI SOSIOLOGI PERNIKAHAN MUBARAK DI PONDOK PESANTREN HIDAYATULLAH MAKASSAR. Jurnal Sosialisasi: Jurnal Hasil Pemikiran, Penelitian Dan Pengembangan Keilmuan Sosiologi Pendidikan, 1, 1-8.

Muhammad, S., Agustang, A., \& Samad, S. (2015). SOCIAL MOVEMENT IN A MINING PROJECT: A STUDY OF SOROWAKOAN CONTENTIOUS POLITICS TOWARDS PT VALE INDONESIA, TBK. IN SOROWAKO, SOUTH SULAWESI. International Journal of Academic Research, 7(Issue ID: IJ15V7N3).

Muis, I., Agustang, A., \& Adam, A. (2020). Lansia Miskin: Demografi Sosial, Distribusi Pekerjaan, Masalah Kesehatan \& Perlindungan Sosial.

Mukti, I., Oruh, S., \& Agustang, A. (2021). Efek Pemberitaan Kekerasan Di Media Sosial Terhadap Citra Negatif Kota Makassar. JISIP (Jurnal Ilmu Sosial Dan Pendidikan), 5(4).

Muslimin, A., Idris, R., \& Agustang, A. (2015). CHILDREN AND SOCIAL ENVIRONMENT OF EDUCATION. International Journal of Academic Research, 7.

Mustadjar, M., \& Agustang, A. (2020). Pergeseran Pola Interaksi Sosial (Studi Pada Masyarakat Banggae Kabupaten Majene). Phinisi Integration Review, 3(2), 138-149.

Nismawati, N., Oruh, S., \& Agustang, A. (2021). Eksistensi Tari Kabasaran Pada Masyarakat Minahasa. Jurnal Ilmiah Mandala Education, 7(4).

Nonci, N., Salman, D., \& Agustang, A. (2014). SILARIANG: STUDY OF THE SOCIAL CONSTRUCTION OF ETHNIC IN MAKASSAR IN GOWA DISTRICT. International Journal of Academic Research, 6(1).

Nur, S., Andi Agustang, \& Arlin Adam. (2020). Uninhabitable Home: Portraits of Poverty in Cities. Indonesian Journal of Social and Environmental Issues (IJSEI), 1(3 SE-), 234-238. https://doi.org/10.47540/ijsei.v1i3.81

Nurbaety, A., Torro, S., \& Agustang, A. (2015). REDUKSI PERAN GOLONGAN BANGSAWAN BUGIS DALAM KEHIDUPAN SOSIAL DI DESA SANREGO KECAMATAN KAHU. FIS.

Nurdin, N., Salman, D., Agustang, A., \& Malago, J. (2021). The Effect of Social Support and Gratitude on Resilient Persons with Disabilities in Makassar City. IOSR Journal of Humanities And Social Science (IOSR-JHSS), 12(5), 14-21.

Nursafitra, M., Agustang, A., Idkhan, A. M., \& Nurwiwiana. (2021). Pengaruh Disiplin Kerja dan Komitmen Organisasi Terhadap Kinerja Pegawai di Kantor Dinas Kependudukan dan Pencatatan Sipil Kabupaten Enrekang. Journal Governance and Politics (JGP), 1(2 SEArticles). https://jurnal.iyb.ac.id/index.php/jgp/article/view/203

Nursinah, Agustang, A., Adam, A., \& Bastiana. (2021). RELAPSE BEHAVIOR OF NAPZA ABUSE AFTER REHABILITATION IN MAKASSAR CITY. PalArch's Journal of 
Archaeology of Egypt/Egyptology, 18(08), 1845-1854.

Oruh, S. (2021). Analisis faktor Keaktifan Kader dalam Kegiatan Posyandu. Jurnal Ilmiah Kesehatan Sandi Husada, 10(1), 319-325.

Oruh, S., \& Agustang, A. (2019). Pengaruh Pengetahuan Keluarga, Stigma Masyarakat dan Kepatuhan Minum Obat Terhadap Kekambuhan Penyakit Gangguan Jiwa di Kota Makassar. 53(9). https://doi.org/10.1017/CBO9781107415324.004

Oruh, S., \& AGUSTANG, A. (2019). PENGARUH PENGETAHUAN KELUARGA, STIGMA MASYARAKAT DAN KEPATUHAN MINUM OBAT TERHADAP KEKAMBUHAN PENYAKIT GANGGUAN JIWA DI KOTA MAKASSAR.

Oruh, S., Agustang, A., \& Bagu, A. A. (2017). KEJADIAN HEROPNAM GANGGUAN JIWA DAN FAKTOR YANG MEMPENGARUHI PADA RUMAH SAKIT KHUSUS DAERAH DADI PROVINSI SULAWESI SELATAN.

Oruh, S., Agustang, A., \& Bagu, A. A. (2020). KEJADIAN HEROPNAM GANGGUAN JIWA DAN FAKTOR YANG MEMPENGARUHI PADA RUMAH SAKIT KHUSUS DAERAH DADI PROVINSI SULAWESI SELATAN.

Oruh, S., \& Nur, H. (2021). Perilaku Masyarakat Pesisir di Hutan Mangrove Desa Balangdatu Kabupaten Takalar. Phinisi Integration Review, 4(2), 292-300.

Oruh, S., Theresia, M., \& Agustang, A. (2019). KESEJAHTERAAN PSIKOLOGIS. Researchgate.Net.

Pettalongi, A., Idris, R., Kasnawi, T., \& Agustang, A. (2015). PROMOTING SOCIAL VALUES IN BUILDING SOCIAL INTERACTION AMONG INTER-ETHNICAL STUDENTS IN SMA KARUNA DIPA, PALU. International Journal of Academic Research, 7.

Rachim, A., Lolo, T. R. A., \& Agustang, A. (2017). RELATIONS AUTHORITY.

Rahman, B., Agustang, A., \& Samad, S. (2015). HEGEMONIC SOCIAL RELATION, A STUDY OF SOCIOLOGY ON OUTSOURCING PRACTICE. International Journal of Academic Research, 7.

Rahman, N., Agustang, A., Akib, H., \& Tahmir, S. (2015). ANALYSIS OF THE DETERMINANT FACTORS OF IN THE LEGISLATIVE PROCESS OF REGIONAL HOUSE OF PEOPLES'REPRESENTATIVES IN TAKALAR REGENCY. International Journal of Academic Research, 7.

RAHMAN, S. (2014). Industri Pabrik dan Masyarakat Lokal di Pinggiran Kota Makassar: Kasus Dua Komunitas Desa Sekitar PT. Kawasan Industri Makassar,(dibimbing oleh HM Idrus Abustam, Andi Agustang dan H. Heri Tahir). universitas negeri makassar.

Ramlafatma, R., Oruh, S., \& Agustang, A. (2021). Efektivitas Pendidikan Moral Dalam Pembentukan Karakter Siswa Di TK Islam Terpadu Asa Sumbawa. Jurnal Ilmiah Mandala Education, 7(4).

Rasyid, D., Agustang, A., Syukur, M., Usman, S., \& Marzuki, M. (2021). Social Transformation of Indonesian Older Adults in Suburbs: An Exploratory-descriptive Qualitative Study. Ethno Med, 15((1-2)), 44-52.

Rasyid, R., Agustang, A., Agustang, A. T. P., Bastiana, B., \& Najamuddin, N. (2020). Analisis Faktor Yang Mempengaruhi Status Kemiskinan Rumah Tangga Pada Wilayah Central Bussiness District (CBD) di Kota Makassar. Majalah Geografi Indonesia, 34(1), 43-52.

Rieuwpassa, S., Agustang, A., \& Adam, A. (2020). Sustainable Peace Development In Ambon (An Analysis Study In Negeri Hila And Dusun Hila Tanah Putih). INTERNATIONAL JOURNAL 
OF SCIENTIFIC \& TECHNOLOGY RESEARCH, 9(2), 477-480.

Risal, R., Agustang, A., \& Syukur, M. (2021). Peranan Perempuan Tani dalam Meningkatkan Ekonomi Keluarga di Kelurahan Bonto Langkasa Kecamatan Bissappu Kabupaten Bantaeng. Phinisi Integration Review, 4(2), 282-291.

Rongrean, B., Abustan, I., \& Agustang, A. (2015). THE SOCIAL DYNAMICS OF PEDICAB DRIVER TOWARD CLIMATE CHANGES IN MAKASSAR. International Journal of Academic Research, 7.

Rusli, M., Bulkis, S., \& Agustang, A. (2018a). MAKNA PERAN DAN MODAL SOSIAL PEREMPUAN NELAYAN BAJO (Studi Fenomenologi di Pesisir Pantai Soropia Kabupaten Konawe). UNIVERSITAS NEGERI MAKASSAR.

Rusli, M., Bulkis, S., \& Agustang, A. (2018b). Usage of Social Capital in Supporting the Welfare of the Coastal Community. IOSR Journal Of Humanities And Social Science (IOSR-JHSS), 23(Issue 8, Ver. 2), 26-32.

Saeni, R., \& Agustang, A. (2014). INDUSTRIAL FACTORY AND LOCAL COMMUNITIES ON THE MARGINAL CITY OF MAKASSAR. International Journal of Academic Research, 6(4).

Safar, M., Agustang, A., \& Adam, A. (2017). PSEUDO AKTOR POLITIK (Studi Genealogi Kekuasaan di Sulawesi Tenggara Indonesia). Pascasarjana.

Sahabuddin, A., Agustang, A., \& Idkhan, A. M. (2021). Fungsi Leadership dalam Peningkatan Pelayanan Publik di Kantor Kecamatan Makassar. Journal Governance and Politics (JGP), 1(2 SE-Articles). https://jurnal.iyb.ac.id/index.php/jgp/article/view/207

Sahabuddin, E., Agustang, A., Manda, D., \& Oruh, S. (2020). Partisipasi Sosial Dalam Pemenuhan Activity Daily Living (Adl) Orang Dengan Gangguan Jiwa Di Kota Makassar. Phinisi Integration Review, 3(2).

Salmah, A. U., Idris, R., Agustang, A., \& Maidin, A. (2013). Socio-Cultural Transformation of Early Detection on Breast Cancer Suspect in Wahidin iSudirohusodo Hospital, Makassar Indonesia.

Samanlangi, A. I., Agustang, A., Adam, A., \& Alim, A. (2021). Disconnectivity Social of Conflict in the Circle of Iron Ore Mine in Bone Regency, South Sulawesi, Indonesia. International Journal of Criminology and Sociology, 10, 963-973.

Sanusi, D., Agustang, A., \& Idkhan, A. M. (2021). Pengembangan Sumber Daya Manusia Dalam Meningkatkan Pelayanan Akademik Di Universitas Puangrimaggalatung Sengkang

Journal Governance and Politics (JGP), $1(2$ SE-Articles). https://jurnal.iyb.ac.id/index.php/jgp/article/view/205

Sapulette, A. A., Agustang, A., \& Syukur, M. (2018). Actor Construction in the Frame of Diversity to Achieve Social Harmony in the Life of Tamilouw Village Society Seram Island-Maluku. IOSR Journal Of Humanities And Social Science (IOSR-JHSS), 23(Issue 2, Ver. 4), 54-60.

Suardi, Agustang, A., \& Jumadi. (2020). A., \& Jumadi.(2020b). The Symbolic Violence Towards Private School Students Through The Frame Stereotype In Bantaeng District Of Indonesia. The International Conference on Innovations in Social Sciences and Education, 578-588.

Suardi, Agustang, A., \& Jumadi. (2021a). DOMINATION OF THE POWER OF QUALITY AND QUANTITY OF STATE SCHOOL EDUCATION AGAINST PRIVATE SCHOOLS AS FACTORS CAUSING SYMBOLIC VIOLENCE OF STUDENTS IN BANTAENG DISTRICT. PROCEEDING 1st INTERNATIONAL CONFERENCE (On Health, Education, and Computer Science Technology), 1(pertama), 246-259. 
Suardi, S., Agustang, A., \& Jumadi, J. (2021b). Dominasi Sekolah Negeri Terhadap Sekolah Swasta Sebagai Penyebab Kekerasan Simbolik Terhadap Siswa Sekolah Swasta. Jurnal Ilmiah Muqoddimah: Jurnal Ilmu Sosial, Politik Dan Hummanioramaniora, 5(2).

Sulaiman, U., Idris, R., Ardi, M., \& Agustang, A. (2014). The Pattern of Cooperation and Poorness: A Seaweed Farming Community in Indonesia. International Journal of Academic Research, 6(5), 239-242.

Sumai, S., Agustang, A., Adam, A., \& Obie, M. (2020). Resilience in Children of Drug Abuse Families: A Case in Pattingalloang, Makassar City, Indonesia.

Sumitro, S., Oruh, S., \& Agustang, A. (2021). Eksistensi Nilai Sosial Budaya Ndeu Paki Oi Mbaru Pada Masyarakat Donggo Kabupaten Bima. Jurnal Ilmiah Mandala Education, 7(4).

Suparman, Oruh, S., \& Agustang, A. (2021). Dinamika Sosial Kelompok Tani (Studi Kasus Petani Bawang Merah Desa perangian Kecamatan Baraka Kabupaten Enrekang). JIIP-Jurnal Ilmiah Ilmu Pendidikan, 4(6), 406-414.

Sutrisno, S., \& Agustang, A. (2018). Peranan Pengawas Sekolah Terhadap Pelaksanaan Kurikulum 2013 Di SMA Negeri 17 Makassar. Jurnal Sosialisasi: Jurnal Hasil Pemikiran, Penelitian Dan Pengembangan Keilmuan Sosiologi Pendidikan, 100-105.

Syaharuddin, S., Agustang, A., Idkhan, A. M., \& Rifdan, R. (2021). STRATEGI DINAS SOSIAL DALAM PENANGANAN ANAK JALANAN DI KOTA MAKASSAR. JISIP (Jurnal Ilmu Sosial Dan Pendidikan), 5(4).

Syamsul, A. (2014). Remaja penjajah seks komersial kota Makassar. Skripsi Sosiologi, Fakultas Ilmu Sosial, Universitas Negeri Makassar, yang di bimbing oleh. Firdaus W. Suhaeb sebagai pembimbing satu dan Andi Agustang sebagai pembimbing dua. universitas negeri makassar.

Syamsul, B., Idrus, A., Jumadi, S., \& Edwar, P. (2014). “A'PA ALLIRI”: THE SOCIAL FUNCTION OF LOCAL INSTITUTION AT MATAJANG SOCIETY. INTERNATIONAL JOURNAL of ACADEMIC RESEARCH, 6(6), 121-125.

Syukurman, S., Agustang, A., \& Adam, A. (2020). Peluang dan Tantangan Lembaga Pendidikan Islam (Ditinjau dari Sosiologi Agama di Desa Doridungga). EDU SOCIATA (Jurnal Pendidikan Sosiologi), 3(2), 71-87.

Thamrin, M., Agustang, A., Adam, A., \& Alim, A. (2021). Disruption of Stunting Social Capital in South Sulawesi, Indonesia (Case Study on 1000FDL Families in Bone and Enrekang Districts). Indian Journal of Public Health Research \& Development, 12(2).

Tinri, M. D. N. (2018). Hotels and Restaurant Tax Revenues Management.

Triono, T., Agustang, A., Idkhan, A. M., \& Rifdan, R. (2021). Motivasi Kerja Pegawai Dalam Pelayanan Publik. JISIP (Jurnal Ilmu Sosial Dan Pendidikan), 5(4).

Ufie, A., Oruh, S., \& Agustang, A. (2021). Maintaining Social Harmony Through Historical Learning Based on Local Wisdom of Indigenous Peoples in Maluku. Historia: Jurnal Pendidik Dan Peneliti Sejarah, 5(1), 31-40.

UMAR, S. (2014). Pola Kerjasama dan Ketidaksejahteraan Komunitas Petani Rumput Laut di Kecamatan Ujung Loe Kabupaten Bulukumba (Dibimbing oleh Promotor Rabihatun Idris serta Kopromotor Muhammad Ardi dan Andi Agustang). universitas negeri makassar.

Urbach, N., \& Agustang, A. (2019). PREFERENSI ORANG TUA DALAM MENYEKOLAHKAN ANAKNYA DI DESA AMOLA KECAMATAN BINUANG KABUPATEN POLEWALI. JURNAL SOSIALISASI, 89-94.

Usman, A., Agustang, A., \& Idkhan, A. M. (2021). Penerapan Pelayanan Prima di Rumah Sakit 
Paru BBPM Makassar. Journal Governance and Politics (JGP), 1(2 SE-Articles). https://jurnal.iyb.ac.id/index.php/jgp/article/view/206

Wardah, S., Salman, D., Agustang, A., \& Fahmid, I. M. (2017). The Contestation of Organic and Non-Organic Agricultural Knowledge in Sustainable Agriculture. Mediterranean Journal of $\begin{array}{llll}\text { Social } & \text { Sciences, } & 8(2 & \text { SE-Articles }),\end{array}$ https://www.richtmann.org/journal/index.php/mjss/article/view/9883

Wardiani, I., \& Agustang, A. (2018). Dampak Pekerjaan Orang Tua Pengrajin Emas Terhadap Pendidikan Anak di Kelurahan Borong Kecamatan Manggala Kota Makassar. UNIVERSITAS NEGERI MAKASSAR.

Wihdania, W., \& Agustang, A. (2020). Pernikahan Tolotang Di Kelurahan Amparita Kecamatan Tellu Limpoe Kabupaten Sidenreng Rappang. Phinisi Integration Review, 3(2), 165-172.

WULAN, S. (2014). Peranan Media Pembelajaran Dalam Meningkatkan Motivasi Belajar Sosiologi Pada Siswa SMA PGRI Galesong. Skripsi. Dibimbing oleh Andi Agustang dan Hj. Musdaliah Mustadjar Program Studi Pendidikan Sosiologi. Fakultas Ilmu Sosial, Universitas Negeri Makassar. universitas negeri makassar.

Yakobus, I. K., Kasnawi, T., Agustang, A., \& Gani, H. A. (2019). Hybridization of the Poso Conflict Resolution Discourse.

Yudhar, A. N., Agustang, A., \& Sahabuddin, J. (2021). Habituation of character values in junior high school students. Cypriot Journal of Educational Sciences, 16(2), 659-668.

Yusuf, M., Agustang, A., Idkhan, A. M., \& Rifdan, R. (2021). Transformasi Lembaga Koperasi Di Era Industri 4.0. JISIP (Jurnal Ilmu Sosial Dan Pendidikan), 5(4). 